\title{
SF3B1 and EIF1AX mutations occur in primary leptomeningeal melanocytic neoplasms; yet another similarity to uveal melanomas
}

Heidi V. N. Küsters-Vandevelde ${ }^{1 *}$, David Creytens ${ }^{2}$, Adriana C. H. van Engen-van Grunsven ${ }^{3}$, Marcel Jeunink', Veronique Winnepenninckx ${ }^{4}$, Patricia J. T. A. Groenen ${ }^{3}$, Benno Küsters ${ }^{3,4}$, Pieter Wesseling ${ }^{1,3,5}$, Willeke A. M. Blokx ${ }^{3}$ and Clemens F. M. Prinsen ${ }^{1}$

\begin{abstract}
Introduction: Like uveal melanomas, primary leptomeningeal melanocytic neoplasms (LMNs) frequently carry GNAQ and GNA11 mutations. However, it is currently unknown whether these LMNs harbor mutations in BAP1, SF3B1 and/or EIF1AX like uveal melanomas as well. In this study, we used Sanger sequencing for the detection of mutations in SF3B1 (hotspots in exon 14 and 15) and EIFIAX (exon 1 and 2 and flanking intronic regions) in a series of 24 primary LMNs. Additionally, BAP1 immunohistochemistry was used as a surrogate marker for the detection of inactivating mutations in the BAP1 gene.

Results: Mutations in either SF3B1 or EIFIAX were identified in 8 out of 24 primary LMNs (33\%). The presence of these mutations was mutually exclusive and occurred in primary LMNs of different malignancy grades (melanocytomas, intermediate-grade melanocytic tumors, melanomas). Complete absence of nuclear BAP1 staining as is typically seen in BAP1-mutated tumors was not observed.

Conclusions: Our finding that an SF3B1 or EIFIAX mutation is present in a substantial subset of primary LMNs underscores that these tumors genetically resemble uveal melanoma and are different from cutaneous melanoma at the genetic level. This information may not only aid in the differential diagnosis of primary versus metastatic melanocytic tumor in/around the central nervous system, but also in the identification of more promising therapeutic approaches targeting the molecular pathways involved in the oncogenesis of LMNs. As none of the primary LMNs in our series showed complete loss of nuclear BAP1 protein, it is unlikely that BAP1 mutations are frequent in these tumors but the role of this gene warrants further investigation.
\end{abstract}

Keywords: Leptomeningeal melanocytic neoplasms, Melanocytoma, Melanoma, Central nervous system, Neurocutaneous melanocytosis, BAP1, SF3B1, EIFIAX

\section{Introduction}

Primary leptomeningeal melanocytic neoplasms (LMNs) are infrequent tumors, forming a spectrum ranging from benign or low-grade malignant melanocytomas to frankly malignant melanomas [1]. These tumors share molecular features with uveal melanomas (UMs). In contrast to cutaneous melanomas (CMs), both

\footnotetext{
* Correspondence: h.kusters@cwz.nl

${ }^{1}$ Department of Pathology, Canisius Wilhelmina Hospital, P.O. Box

90156500GS, Nijmegen, The Netherlands

Full list of author information is available at the end of the article
}

LMNs and UMs frequently carry mutations in the $\mathrm{G}$ protein encoding genes GNAQ and GNA11, whereas mutations in BRAF and in the TERT promoter are infrequent [2-8]. This situation reflects the heterogeneous molecular background of different groups of melanoma and has important implications for targeted therapy.

In the past years, inactivating mutations in the tumor suppressor gene BAP1 (BRCA-associated protein 1) were shown to be implicated in UM $[9,10]$. The BAP1 gene is located on chromosome 3p21.1 and encodes a nuclear 
ubiquitinase involved in epigenetic modulation of chromatin [11]. Somatic BAP1 mutations are predominantly present in UMs with monosomy 3 ( $\sim 85 \%)$, the latter being a strong predictor for metastatic disease $[9,10,12]$. In this setting BAP1 functions as a tumor suppressor gene, with loss of one copy of chromosome 3 and mutation in the other $B A P 1$ allele representing the two hits causing inactivation of this gene. Indeed, in UMs with disomy 3 (and a good prognosis), mutations in BAP1 are rare $[6,9,10]$. A small proportion of patients with UM ( 2-3\%) harbor a germline mutation in BAP1 [13]. These patients suffer from the $B A P 1$ hereditary cancer syndrome and have an increased risk of developing cutaneous melanocytic tumors as well as a spectrum of non-melanocytic neoplasms including mesothelioma, renal cell carcinoma, meningeoma, and adenocarcinoma of the lung [14, 15]. Very recently, it was suggested that primary leptomeningeal melanoma is part of this cancer predisposition syndrome as well [16].

Furthermore, recurrent hotspot mutations in the SF3B1 gene (mainly at codon 625) and mutations of the EIF1AX gene (spread over exon 1 and 2) were recently reported in UMs, especially in tumors with disomy 3 (up to 30 and $50 \%$ of disomy 3 tumors, respectively) [17-19]. These mutations in UMs appeared to be largely mutually exclusively with $B A P 1$ mutations, while in CMs these mutations were found to be very infrequent $(\sim 1 \%)$ [20].

It is currently unknown whether somatic mutations in $B A P 1, S F 3 B 1$ and $E I F 1 A X$ also characterize primary LMNs. Using Sanger sequencing, we searched for mutations in hotspot regions of SF3B1 (exon 14 and 15) and exon 1 and 2 and flanking intronic regions of EIF1AX in a series of 24 primary LMNs. Additionally, we performed immunohistochemistry for the detection of BAP1 protein loss as a surrogate marker for identification of inactivating $B A P 1$ mutations.

\section{Materials and methods}

\section{Patients and histopathology}

Formalin fixed and paraffin embedded (FFPE) tissue samples of twenty-four primary LMNs were obtained through the Dutch nationwide histopathology and cytopathology data network and archive (PALGA) [21]. Histology was revised by HK; WHO 2007 criteria were used for classification into melanocytoma, intermediate-grade melanocytic tumor and melanoma [1]. Information on mutation status of GNAQ, GNA11, BRAF, NRAS and chromosome 3 status of cases \#1-7, \#10, \#13-18, and $\# 22-24$ has been published previously [2, 22, 23]. The study was approved by the local ethics committees of the Canisius Wilhelmina Hospital (ref.nr. LTC/TT/0132015) and Radboud University (CMO, ref.nr/Dossiernummer: 2015-1692).

\section{DNA extraction}

Representative regions of about five 4- $\mu$ m-thick FFPE sections with an estimated tumor cell percentage of at least $70 \%$ were manually dissected and used for DNA extraction. After heating in ATL buffer, the tissue sections were incubated in proteinase $\mathrm{K}$ for one hour, followed by subsequent purification of the DNA according to the manufacturer (QIAamp DNA Mini Kit, QIAGEN GmbH, Germany). After DNA purification, possible melanin contamination was removed by using an inhibitor removal kit (OneStep ${ }^{\text {TM }}$ PCR Inhibitor Removal Kit, Zymo Research). The DNA sample concentration was assessed spectrophotometrically (Varian Cary 50 spectrophotometer, Agilent Technologies). The integrity of the isolated DNA was tested by means of a multiplex ladder PCR [24].

\section{BAP1 immunohistochemistry}

Immunohistochemistry was performed on 4- $\mu$ m-thick FFPE sections using an automated immunostainer (Benchmark XT, Ventana Medical Systems, Tucson, AZ, USA) according to the manufacturer's instructions. Sections were immunostained with a primary monoclonal antibody against BAP1 (clone C4, 1:100 dilution, Santa Cruz Biotechnology, Dallas, TX, USA) using 3,3-diaminobenzidine (DAB) as chromogen. Selected cases that showed a staining result that was difficult to interpret including strongly pigmented tumors (patients \#1-8, \#12-16, \#22-24) were manually stained (clone C4, 1:50 dilution, Immunologic, Duiven, the Netherlands) according to the manufacturer's instructions. The VECTOR NovaRED Peroxidase (HRP) Substrate Kit was used for visualization (Vector Laboratories, USA, Catalogue Number SK-4800). Nuclei of endothelial and lymphocytic cells in the slides served as positive internal control for BAP1 protein expression. The staining results were scored independently by three pathologists (HK, DC, WB). The percentage of positive tumor cell nuclei was scored only in areas with positive internal controls.

\section{Mutation analysis}

Sanger sequencing was used for analysis of mutations in hotspot areas of SF3B1 including codon 625 and codon 700 as well as for mutations in exon 1 and 2 and flanking intronic regions of EIF1AX. Primers are listed in Table 1. Normal tissue to exclude germline mutations was not available.

All primers contained a M13 forward or M13 reverse consensus sequence for sequencing. PCR amplification was performed in a total volume of $25 \mu \mathrm{l}$, containing 20 ng DNA, PCR Reaction Buffer with $20 \mathrm{mM} \mathrm{MgCl} 2$ (Roche), $200 \mu \mathrm{M}$ of each deoxynucleotide triphosphate, 10 pmol of each primer and 2 units of FastStart Taq DNA polymerase (Roche). DNA amplification was performed in a Biometra T-Professional thermocycler (Westburg). The PCR was started with $5 \mathrm{~min}$. at $95{ }^{\circ} \mathrm{C}$ 
Table 1 Primers used for mutational analysis

\begin{tabular}{llll}
\hline Gene & Exon & $\begin{array}{l}\text { Forward (Fw) } \\
\text { Reverse (Rv) }\end{array}$ & Primer sequence 5'- 3' \\
\hline SF3B1 & 14 & Fw & TGATATGGAAAGAAATGGTTGAAG \\
& & Rv & AGGGCAATAAAGAAGGAATGC \\
SF3B1 & 15 & Fw & TGCAGTTTGGTTAATAGTTG \\
& & Rv & GGCCAAAGCACTGATGGT \\
EIF1AX & 1 & Fw & CCCCTCGGAGCAGCAG \\
& & Rv & CCTGGGTGACCTGCAATCTAC \\
EIF1AX & 2 & FW & GCCTTAATTCATTTATTTCATACTGTT \\
& & Rv & AGGATGTTATTTAAAAAGCGTAATT
\end{tabular}

and followed with 40 cycles of denaturation $30 \mathrm{~s}$ at $95{ }^{\circ} \mathrm{C}$, annealing at $60{ }^{\circ} \mathrm{C}$ for $45 \mathrm{~s}$ and extension at $72{ }^{\circ} \mathrm{C}$ for $30 \mathrm{~s}$, followed by a final extension at $72{ }^{\circ} \mathrm{C}$ for $10 \mathrm{~min}$. and cooling down for $5 \mathrm{~min}$. at $22{ }^{\circ} \mathrm{C}$. All PCR products were purified with ExoSAP-IT (Affymetrix). Two $\mu$ of the PCR product was used for the sequence reaction on an $\mathrm{ABI}$ PRISM 3500XL DNA analyzer (Applied Biosystems). Both strands were sequenced using the M13 primers.

Information on $B A P 1$ mutation status was available for three patients (\#20, \#23, \#24) (obtained by Sanger sequencing as previously described) while the chromosome 3 status was in part obtained from a previous MLPA study [22, 25]. For all cases, the mutation status of GNAQ and GNA11 (codons 209 and 183), BRAF (codon 600) and NRAS (codons 12, 13 and 61) was available, part of this information has been published previously [2, 22, 23].

\section{Results}

\section{Patients and histology}

Main patient characteristics are summarized in Table 2. The study group consisted of thirteen melanocytomas, seven intermediate-grade melanocytic tumors and four primary leptomeningeal melanomas. A subset of the tumors was strongly pigmented.

All patients were adults, the age at operation ranging from 27-69 years. None of the patients had clinical evidence of primary melanoma outside the CNS. One patient was known with a large congenital melanocytic nevus in the buttocks in the context of neurocutaneous melanocytosis (patient \#21). Two of the patients in this series developed liver metastases, both after an initial histological diagnosis of intermediate-grade melanocytic tumor (patients \#19 and \#20). One melanoma patient developed distant metastases to bones and lungs (patient \#23).

\section{BAP1 immunohistochemistry}

BAP1 immunohistochemistry was available in 20 out of 24 cases. For three cases there was no FFPE material left for immunohistochemistry (patients \#9-11), while in one patient the tumor showed intense pigmentation, preventing reliable interpretation of staining results (patient \#6). Only nuclear staining was considered as positive, although cytoplasmic staining was found in a substantial subset of tumors as well. In two melanocytomas (patients \#1 and \#4), a mosaic pattern with alternating positive and negative (areas of) nuclear staining was present, the vast majority (about $80 \%$ ) of these nuclei being positive (Fig. 1a, b). All other cases showed moderate to strong nuclear staining in $90 \%$ or more of nuclei. Complete absence of nuclear BAP1 staining as is typically seen in BAP1-mutated tumors was not observed (Figs. 1, 2 and 3).

\section{Mutation analysis}

We detected a total of three mutations in SF3B1 (13\%), including two hotspot mutations at codon 625 (R625C and $\mathrm{R} 625 \mathrm{H})$ and one mutation affecting codon 634 (V634I) (Table 2). Mutations in codon 700 of SF3B1 were not detected. In the cosmic database the V634I mutation in SF3B1 (c.1900G > A (p.(Val634Ile))) has not been reported in cancer before (http://cancer.sanger.ac.uk/cos mic). This mutation was present in a GNAQ-mutated melanocytoma of a 27-year-old male patient who developed leptomeningeal seeding shortly after initial presentation (patient \#4) (Fig. 1a, b, e).

One case with mutation in codon 625 of SF3B1 (c.1873C > T (p.(Arg625Cys))) concerned an intermediategrade melanocytic tumor showing aggressive behavior with leptomeningeal seeding (patient \#18). A concomitant GNA11 mutation was present in this tumor while immunohistochemistry showed intact nuclear BAP1 expression (Fig. 1c, d, f).

The other mutation in codon 625 of SF3B1 (c.1874G > A (p.(Arg625His))) was present in a CNS melanoma of a 31-year-old woman (patient \#21). Since birth this patient was known with a large congenital melanocytic nevus on the buttocks for which multiple excisions had been performed, confirming melanocytic nevus without malignant transformation. At the age of 29, she developed an intracranial tumor in the left frontal region with diffuse leptomeningeal extension. Biopsy showed melanoma in a background of diffuse melanocytosis, consistent with the diagnosis of primary melanoma of the CNS occurring in the context of neurocutaneous melanocytosis. This was supported by the presence of an NRAS mutation in both the CNS melanoma and in a more recent biopsy of the melanocytic nevus (c.182A > G (p. (Gln61Arg))). In contrast, the SF3B1 mutation (c.1874G > A (p.(Arg625His))) was present in the CNS melanoma but not in the melanocytic nevus. Immunohistochemically, intact BAP1 protein expression was found in the tumor cell nuclei of this melanoma (Fig. 2).

A point mutation in EIF1AX was detected in five of the primary LMNs in this series (21\%), each leading to 
Table 2 Patient characteristics and results of immunohistochemistry and mutation analyses

\begin{tabular}{|c|c|c|c|c|c|c|c|c|c|c|c|}
\hline Patient & Sex & Age & Diagnosis & Location & $\begin{array}{l}\text { BAP1 } \\
\text { immunostaining }\end{array}$ & Chrom 3 & $\begin{array}{l}\text { SF3B1 exon } 14 \\
\text { (codon } 625 \text { ) }\end{array}$ & $\begin{array}{l}\text { SF3B1 exon } 15 \\
\text { (codon } 700)\end{array}$ & EIFIAX exon 1 & EIFIAX exon 2 & Follow-up \\
\hline 1 & $\mathrm{~F}$ & 27 & $M C$ & Right CPA & $+(80 \%)$ & disomy & wt & wt & $\begin{array}{l}\text { Intronc.1- } \\
4 C>T\end{array}$ & wt & local recurrence \\
\hline $2^{\mathrm{a}}$ & M & 41 & MC & $\mathrm{CO}-\mathrm{C} 3$ & + & disomy & wt & wt & $\begin{array}{l}\text { c. } 9 \mathrm{G}>\mathrm{C} \\
(\text { p.(Lys3Asn)) }\end{array}$ & wt & $\begin{array}{l}\text { local recurrence and LM seeding } 3 \text { years } \\
\text { after diagnosis }\end{array}$ \\
\hline $3^{\mathrm{a}}$ & M & 47 & MC & $\begin{array}{l}\text { Extramedullary, } \\
\text { intradural }\end{array}$ & + & disomy & wt & wt & wt & na & $\begin{array}{l}\text { local recurrence and LM seeding shortly after } \\
\text { initial presentation }\end{array}$ \\
\hline $4^{a}$ & M & 27 & MC & $\begin{array}{l}\text { Tentorium } \\
\text { cerebelli }\end{array}$ & $+(80 \%)$ & na & $\begin{array}{l}\text { c.1900G > A } \\
\text { (p. (Val634lle)) }\end{array}$ & wt & wt & na & LM seeding shortly after initial presentation \\
\hline 5 & M & 38 & MC & C5-6 & + & na & wt & na & na & na & tumor spread in neck and vertebra; deceased \\
\hline $6^{\mathrm{a}}$ & M & 41 & MC & Th6 & na & na & wt & wt & na & na & local recurrence; no distant metastasis \\
\hline $7^{\mathrm{a}}$ & v & 57 & MC & Th11 & + & disomy & wt & wt & wt & wt & unknown \\
\hline $8^{\mathrm{b}}$ & $\mathrm{F}$ & 62 & MC & $\mathrm{CO}-\mathrm{C} 1$ & + & na & wt & wt & wt & wt & unknown \\
\hline 9 & $\mathrm{~F}$ & 23 & MC & Fossa posterior & na & na & wt & wt & wt & wt & local recurrence after 2 years \\
\hline $10^{\mathrm{a}}$ & M & 55 & MC & C3-6 & na & na & wt & wt & wt & na & local recurrence 8 years after diagnosis \\
\hline $11^{\mathrm{a}}$ & M & na & MC & Spinal region & na & na & wt & wt & wt & wt & unknown \\
\hline 12 & M & 69 & MC & $\begin{array}{l}\text { Conus } \\
\text { medullaris }\end{array}$ & + & na & wt & wt & wt & wt & alive \\
\hline 13 & M & 37 & $M C$ & Left CPA & + & na & wt & wt & wt & wt & unknown \\
\hline $14^{\mathrm{b}}$ & $\mathrm{F}$ & 68 & IMT & $\begin{array}{l}\text { Tentorium } \\
\text { cerebelli }\end{array}$ & + & disomy & wt & wt & $\begin{array}{l}\text { c. } 11 A>G \\
(p .(A s n 4 \text { Ser }))\end{array}$ & wt & deceased (not disease related) \\
\hline $15^{\mathrm{a}}$ & $\mathrm{v}$ & 44 & IMT & Cauda & + & disomy & wt & wt & wt & wt & stable (no recurrence) \\
\hline $16^{\mathrm{a}}$ & M & 41 & IMT & $\begin{array}{l}\text { Intramedullary } \\
\text { (NOS) }\end{array}$ & + & disomy & wt & wt & wt & $\begin{array}{l}\text { c.25G }>C \\
\text { (p.(Gly9Arg)) }\end{array}$ & local recurrence and LM seeding \\
\hline $17^{\mathrm{b}}$ & v & 59 & IMT & $\begin{array}{l}\text { Vermis } \\
\text { cerebelli }\end{array}$ & + & na & wt & wt & wt & wt & unknown \\
\hline $18^{\mathrm{b}}$ & $\mathrm{F}$ & 30 & IMT & Th10-11 & + & disomy & $\begin{array}{l}\text { c. } 1873 C>T \\
(p .(\operatorname{Arg} 625 C y s))\end{array}$ & wt & wt & wt & $\begin{array}{l}\text { leptomeningeal seeding; deceased (disease } \\
\text { related) }\end{array}$ \\
\hline $19^{\mathrm{a}}$ & $\mathrm{F}$ & 53 & IMT & Th9 & + & na & wt & wt & wt & $\begin{array}{l}\text { c.28A > G } \\
(p .(\text { Lys } 10 G \mid u))\end{array}$ & liver metastasis shortly after diagnosis \\
\hline $20^{\mathrm{a}, \mathrm{e}}$ & $\mathrm{F}$ & 48 & IMT & $\begin{array}{l}\text { Cervical spinal } \\
\text { region }\end{array}$ & + & disomy & wt & wt & wt & wt & $\begin{array}{l}\text { local recurrence after } 3 \text { years; distant } \\
\text { metastasis to liver and pancreas } 1 \text { year later; } \\
\text { deceased } 5 \text { years after initial presentation }\end{array}$ \\
\hline $21^{c}$ & F & 31 & MM & Frontal left & + & disomy & $\begin{array}{l}\text { c. } 1874 G>A \\
(p .(\operatorname{Arg} 625 \mathrm{His}))^{d}\end{array}$ & wt & wt & & $\begin{array}{l}\text { neurocutaneous melanocytosis patient; the } \\
\text { SF3B1 mutation was only present in the CNS } \\
\text { melanoma and not in the congenital } \\
\text { melanocytic nevus of the skin }\end{array}$ \\
\hline
\end{tabular}


Table 2 Patient characteristics and results of immunohistochemistry and mutation analyses (Continued)

\begin{tabular}{|c|c|c|c|c|c|c|c|c|c|c|c|}
\hline $22^{a}$ & $M$ & 62 & MM & Th7-9 & + & na & na & na & $\begin{array}{l}c .9 G>C \\
(p .(L y s 3 A s n))\end{array}$ & na & unknown \\
\hline $23^{\mathrm{a}, \mathrm{e}}$ & $\mathrm{F}$ & 59 & MM & S2 & + & $\begin{array}{l}\text { Monosomy } \\
3\end{array}$ & wt & $w t$ & wt & wt & $\begin{array}{l}\text { distant metastases after } 2 \text { years (bone, lungs); } \\
\text { liver metastasis unknown }\end{array}$ \\
\hline $24^{\mathrm{a}, \mathrm{e}}$ & M & 55 & $\mathrm{MM}$ & L1-L2 & + & na & wt & wt & wt & wt & $\begin{array}{l}\text { leptomeningeal seeding } 1 \text { year after initial } \\
\text { presentation; no distant metastases; deceased }\end{array}$ \\
\hline
\end{tabular}

Information on GNAQ/GNA11 mutation status and chromosome 3 status of cases 1-7, 10, 13-18, and 22-24 has been published previously [2, 22, 23]

$F$ female, $M$ male, $M C$ melanocytoma, IMT intermediate-grade melanocytic tumor, MM melanoma, LM leptomeningeal, na not available (BAP1 immunohistochemistry of cases \#9-11) or not assessable, CPA

cerebello-pontine angle

+ positive nuclear staining in $90 \%$ or more of nuclei

${ }^{\mathrm{a}} \mathrm{GNAQ}^{\mathrm{C} 209}$ or

${ }^{\mathrm{b}} \mathrm{GNA} 11^{\mathrm{Q} 209}$ or

${ }^{\mathrm{C}} \mathrm{NRAS}^{\mathrm{Q} 61}$ mutation present

${ }^{d}$ the SF3B1 mutation was not present in the congenital melanocytic nevus of this neurocutaneous melanocytosis patient

${ }^{e}$ cases for which all 17 exons of the BAP1 gene were tested with Sanger sequencing and no mutations were detected 


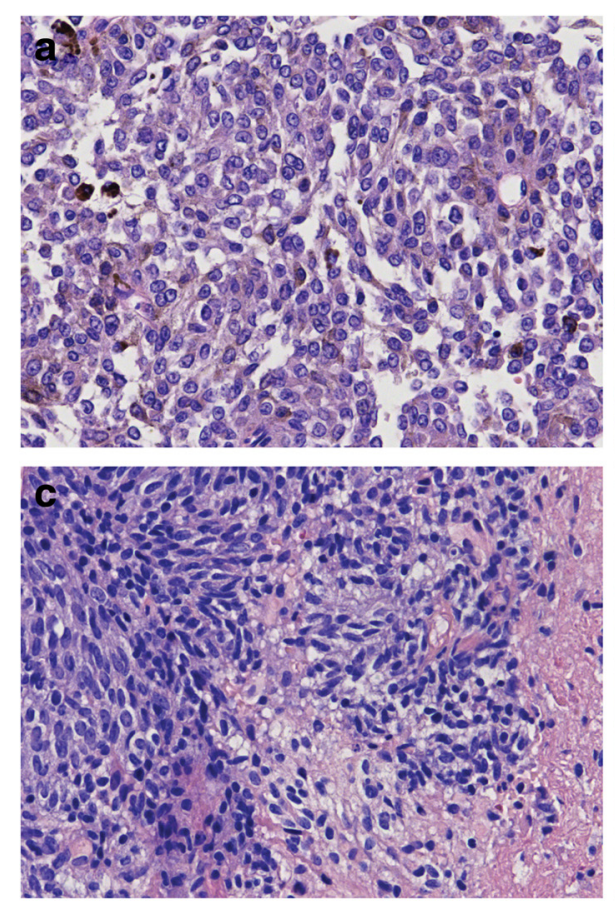

e

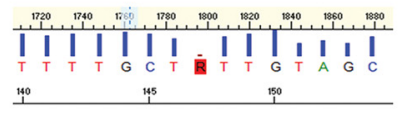

SF3B1 c.1900G>A (p.(Val634lle))

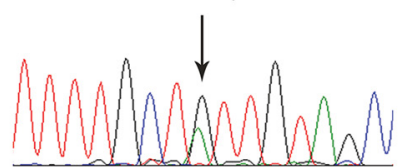

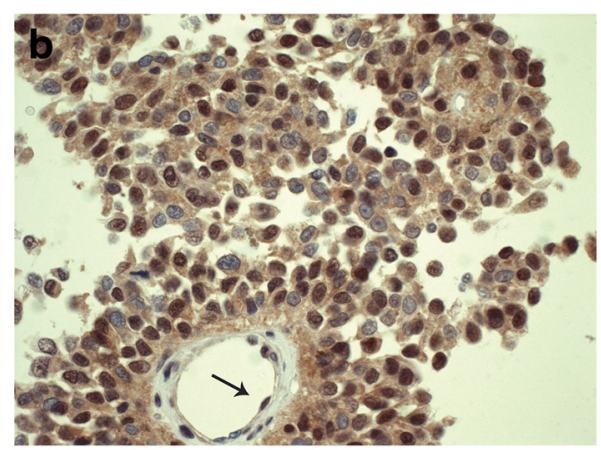

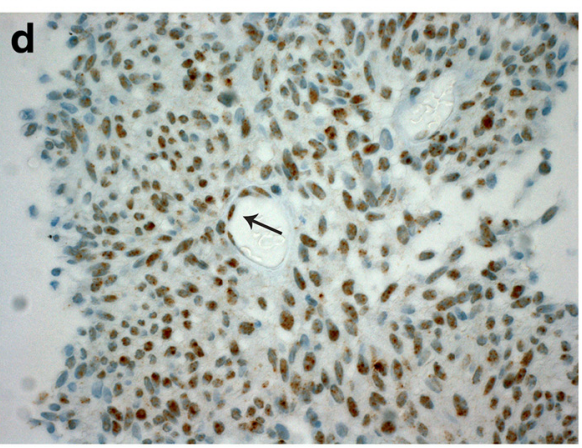

f

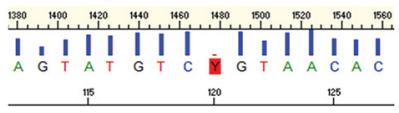

SF3B1 c.1873C>T (p.(Arg625Cys))

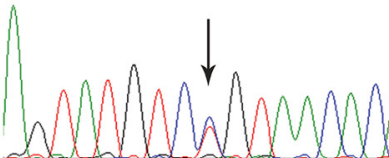

Fig. 1 Examples of SF3B1 mutation in a primary leptomeningeal melanocytoma and intermediate-grade melanocytic tumor. a. Hematoxylin-andEosin (H\&E) staining of the melanocytoma of patient \#4 showing round to oval, slightly pleomorphic, vesicular nuclei, often with a prominent nucleolus, and with dispersed melanin pigment in the cytoplasm of the tumor cells. $\mathbf{b}$. BAP1 immunostaining of this melanocytoma revealed heterogeneous staining of the tumor cell nuclei, the majority of these nuclei being positive. The arrows indicate nuclear staining in endothelial cells. Note that the tumor cells also show variable cytoplasmic staining. c. H\&E staining of the intermediate-grade melanocytic tumor of patient \#18 showing a proliferation of spindle cells with invasion in glial tissue (at the right). $\mathbf{d}$. BAP1 immunostaining of the tumor depicted in (c) reveals positive nuclei in more than $90 \%$ of tumor cells. The arrows indicate nuclear staining in endothelial cells. e. Forward sequence tracing surrounding codon 634 of SF3B1 showing the c.1900G > A (p.(Val634lle)) mutation detected in the tumor depicted in (a) and (b). f. Forward sequence tracing surrounding codon 625 of SF3B1 showing the c.1873C > T (p.(Arg625Cys)) mutation present in the tumor depicted in (c) and (d)

an amino acid substitution (at codons $3,4,9$ or 10). These mutations occurred in tumors diagnosed as melanocytomas, intermediate-grade tumors, and melanomas (Fig. 3). In addition, one point mutation was detected in the Kozak consensus sequence of exon 1 (c.1-4C $>\mathrm{T}$ ) which may have an influence on the start of translation (patient \#1). EIF1AX mutations were mutually exclusive with SF3B1 mutations and generally co-occurred with GNAQ or GNA11 mutations (Table 2).

\section{Discussion}

In this study we investigated whether primary LMNs share genetic alterations with UMs in addition to GNAQ and GNA11 mutations. Recently, a role for BAP1 in primary melanoma of the CNS was suggested based on the identification of a BAP1 germline mutation in a patient with primary CNS melanoma with monosomy 3 and a family history of UM and meningioma [16]. In our study, we chose for BAP1 immunohistochemistry as a surrogate marker for the identification of an underlying inactivating BAP1 mutation as it offers an economical and faster alternative to sequence analysis of all 17 exons of BAP1 [12, 26, 27]. Typically, complete loss of nuclear BAP1 expression is found in BAP1-mutated UMs [12, 26-28]. None of the samples in our series showed such complete loss of nuclear BAP1 staining, suggesting absence of underlying BAP1 mutations. In three cases (including the single patient 

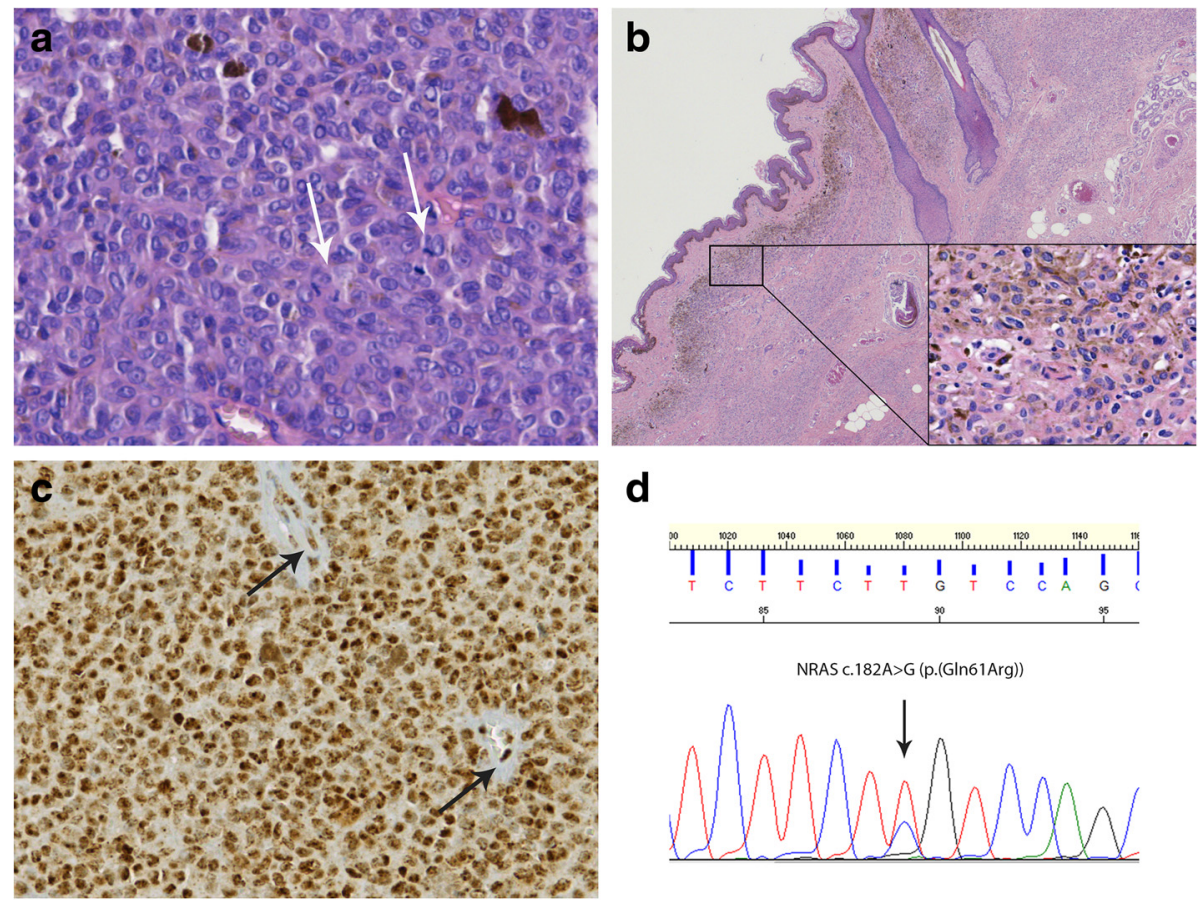

d
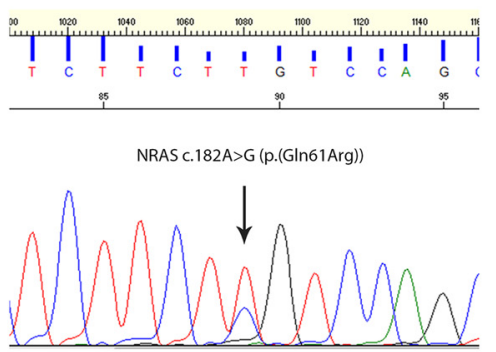

e

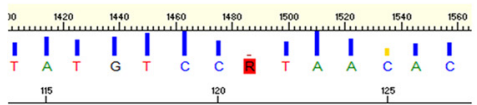

f

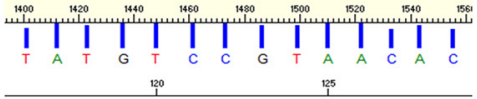

SF3B1 codon $625 \mathrm{wt}$
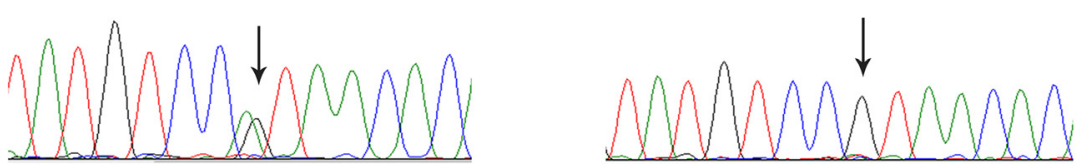

Fig. 2 SF3B1 mutation in primary leptomeningeal melanoma in a patient with neurocutaneous melanocytosis. a. H\&E staining of the primary LMN diagnosed as melanoma; the arrows indicate mitotic figures (patient \#21). b. H\&E staining of the congenital melanocytic nevus of the buttocks; in the right lower corner higher magnification of the superficial part of the nevus with a proliferation of bland nevoid cells. c. BAP1 immunostaining of the melanoma depicted in (a) with nuclear staining in more than $90 \%$ of tumor cells. $\mathbf{d}$. Sequence tracing surrounding codon 61 of NRAS showing a c.182A > G (p.(Gln61Arg)) mutation present in both the CNS melanoma as well as in the congenital melanocytic nevus (reverse sequence/antisense strand). e. Forward sequence tracing surrounding codon 625 of SF3B1 showing a c.1874G > A (p.(Arg625His)) mutation in the CNS melanoma. f. The SF3B1 mutation depicted in (e) is absent in the congenital melanocytic nevus of this patient

with monosomy 3 in the $\mathrm{LMN}$ and the patient with liver metastases but disomy 3 in the LMN), BAP1 mutation status was available through Sanger sequencing analyses and confirmed absence of mutations. However, our study has some limitations. In some cases, BAP1 mutations may still have been missed as BAP1 immunohistochemistry was reported to have a sensitivity of $\sim 88 \%$ [12]. Also, very rarely, heterogeneous ('mosaic') BAP1 nuclear immunostaining has been described in UM cases with a BAP1 mutation showing loss of nuclear staining in only $20 \%$ of nuclei [12]. In our series, two cases showed nuclear BAP1 staining in about $80 \%$ of tumor cells and an underlying BAP1 mutation can thus not completely be ruled out. Furthermore, as BAP1 is mainly implicated in metastatic UM with monosomy 3, there might be a selection bias in our patient group as it mainly concerns (relatively) low-grade tumors with disomy for chromosome 3. A larger number of primary LMNs with monosomy 3 should thus be investigated to further explore the role of BAP1 in these neoplasms. This is also important for therapeutic reasons as epigenetic modulators such as histone deacetylase (HDAC) inhibitors were shown to reverse the biochemical effects of BAP1 mutations in UM cells by inducing growth arrest and differentiation. Clinical trials are now evaluating HDAC inhibitors as a therapeutic option in UM patients $[13,29]$.

Recurrent mutations in the SF3B1 gene have been detected in several types of cancer such as UMs, breast 


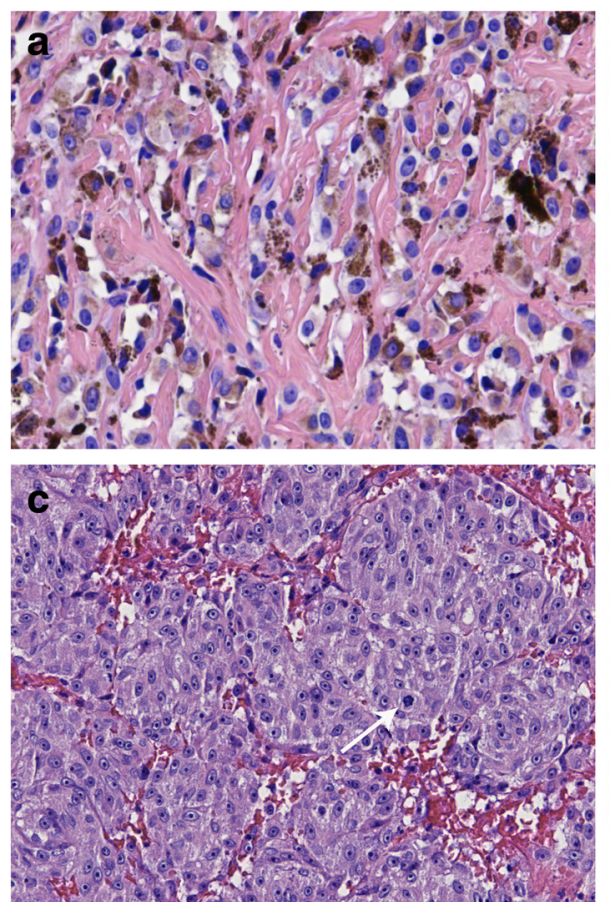

e

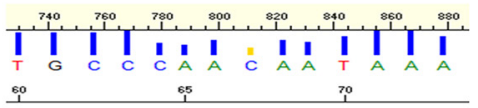

EIF1AX c.9G>C (p.(Lys3Asn))

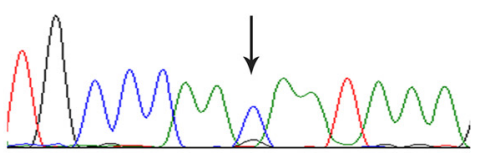

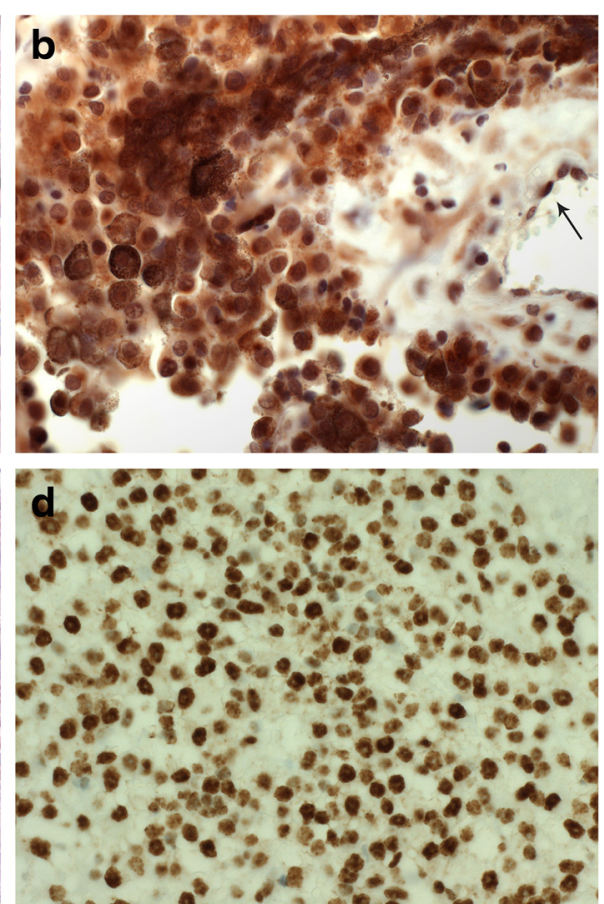

f

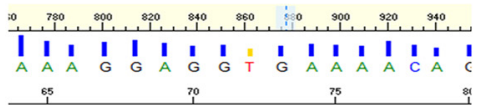

EIF1AX c.28A>G (p.(Lys10Glu))

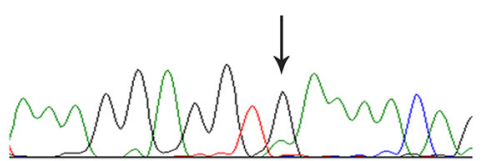

Fig. 3 Examples of EIFTAX mutation in a primary leptomeningeal melanocytoma and intermediate-grade melanocytic tumor. a. H\&E staining of the melanocytoma of patient \#2, consisting of epithelioid cells with moderate to strong melanin pigmentation. $\mathbf{b}$. BAP1 immunostaining of the melanocytoma depicted in A revealing positive staining of nuclei in more than $90 \%$ of tumor cells. The arrows indicate nuclear staining in endothelial cells. Note that cytoplasmic staining is also present. c. H\&E staining of the intermediate-grade melanocytic tumor of patient \#19 showing a nested proliferation of epithelioid cells; the arrow indicates a mitotic figure. $\mathbf{d}$. BAP1 immunostaining of the intermediate-grade melanocytic tumor depicted in C revealing positive staining of nuclei in more than $90 \%$ of tumor cells. e. Forward sequence tracing surrounding codon 3 of EIFTAX showing a c.9G > C (p.(Lys3Asn)) mutation in the melanocytoma depicted in (a) and (b). f. Forward sequence tracing surrounding codon 10 of EIF1AX showing a c.28A > G (p.(Lys10Glu)) mutation in the intermediate-grade melanocytic tumor depicted in (c) and (d)

and pancreatic carcinoma, and hematological diseases like CLL and MDS [18, 30-33]. These mutations affect hotspot codons, the hotspot being associated with cancer type. For example, codon 700 mutations are frequently present in CLL and MDS, while in UMs codon 625 is much more frequently involved [18, 34]. Especially in low-grade UMs with disomy for chromosome 3, heterozygous mutations in SF3B1 are present in 10 to $30 \%$ of UM [6, 17-19]. The fact that in UMs these SF3B1 mutations are almost mutually exclusive with $B A P 1$ mutations suggests different pathways in the oncogenesis and/or malignant progression of these neoplasms. SF3B1 encodes subunit 1 of splicing factor 3b, which is a component of the spliceosome that participates in splicing of pre-mRNA. It was shown that SF3B1 mutations are associated with differential alternative splicing of several protein encoding genes in UMs [19]. Moreover, SF3B1 mutant cell lines were found to be sensitive to the SF3b complex inhibitor spliceostatin A, suggesting a new therapeutic target in tumors carrying this mutation [32]. Up to now, it was unknown whether SF3B1 mutations also occur in primary LMNs. In our series, in three out of 24 cases (13\%) an SF3B1 mutation affecting codon 625 or 634 was detected, this is at the lower end of the range of the frequency of SF3B1 mutations reported in UMs (10-30 \%) and in contrast to the very low frequency reported for CMs $(\sim 1 \%)[6,17-20]$. As far as could be assessed, all three 
LMNs showing an SF3B1 mutation were tumors with disomy for chromosome 3. Furthermore, like in UMs, in two cases the SF3B1 mutation co-occurred with a GNAQ or GNA11 mutation, while in a third case (with neurocutaneous melanocytosis) an NRAS mutation was present. The GNAQ, GNA11 and NRAS genes are now thought to play a role in the initiation of tumorigenesis, while mutation in $S F 3 B 1$ (or BAP1) would then occur in a later phase of the oncogenic process [18]. The cooccurrence of an NRAS and an SF3B1 mutation in the CNS melanoma of the neurocutaneous melanocytosis patient in our study is interesting in this respect as the SF3B1 mutation was absent in the congenital melanocytic nevus of this patient. Patients with neurocutaneous melanocytosis have a large and/or multiple congenital melanocytic nevi of the skin in association with a primary LMN [1]. Instead of GNAQ or GNA11 mutations, these patients frequently demonstrate identical NRAS mutations in both the congenital melanocytic nevus and in the LMN $[35,36]$. This is thought to be the result of an NRAS-mutated clone of melanocyte precursors migrating to skin and CNS early in embryogenesis [37]. The observation that the SF3B1 mutation was not present in the melanocytic nevus of this patient suggests that it indeed plays a role later on in tumorigenesis. In addition, all three mutations in SF3B1 in our study were found in tumors which clinically showed aggressive behavior. However, the number of patients and the follow-up in our study are too limited to allow for firm conclusions about a prognostic role of $S F 3 B 1$ in this setting. Of note, in one of the three patients the SF3B1 mutation (c.1900G > A (p.(Val634Ile))) was present in a neoplasm diagnosed as melanocytoma, suggesting that SF3B1 mutations are not necessarily associated with worrisome histology.

Several recent studies have reported mutations in EIF1AX in different cancer types, including melanoma and thyroid and ovarian cancer [17, 38, 39]. In UMs, heterozygous mutations in exon 1 and 2 of EIF1AX have reported to occur especially in tumors with disomy for chromosome 3 (up to $48 \%$ of these tumors) [17]. Mutations may occur in different loci of these exons and lead to amino acid substitutions or short deletions. Rarely splice site mutations have been reported [6, 10, 17]. EIF1AX encodes the eukaryotic translation initiation factor $1 \mathrm{~A}$ (eIF1A), which is involved in initiation phase of translation of eukaryotic cells by stabilizing the formation of the functional ribosome around the AUG start codon. The exact role of EIF1AX mutations in tumorigenesis is currently not well understood but it has been suggested that mutations in EIF1AX could diminish the rate of bulk translation $[17,40]$. In our series of primary LMNs we found a relatively high frequency of EIF1AX missense mutations $(21 \%)$ which is in the range reported for UM (19-48 \%) [6, 17]. In contrast, EIF1AX mutations are very rare in $\mathrm{CM}(5 / 231, \sim 2 \%)$ [20]. As in UMs, as far as could be assessed in our series, these mutations occurred in primary LMNs with disomy for chromosome 3 and were mutually exclusive with SF3B1 mutations, but co-occurred with GNAQ or GNA11 mutations. In addition (and like SF3B1 mutations), the EIF1AX mutations in our cases occurred both in melanocytomas as well as melanomas, suggesting that they are not necessarily associated with worrisome histology, but the prognostic implications of these mutations remain to be elucidated. Finally, the EIF1AX and SF3B1 mutations in LMNs occurred in hotspot regions of these genes, and in UMs such mutations were shown to be somatic in origin [17, 18]. However, as non-neoplastic tissue of the patients with LMNs was not available for further testing, strictly speaking we cannot rule out the possibility that in some of these cases it concerned a germline mutation.

\section{Conclusions}

We report for the first time that a substantial subset of LMNs carries a mutation in SF3B1 or EIF1AX. Like in UMs, SF3B1 or EIF1AX mutations are mutually exclusive and generally co-occur with either a GNAQ or GNA11 mutation (SF3B1 occasionally with NRAS mutation), suggesting that $S F 3 B 1$ and EIF1AX mutations occur later on in the tumorigenesis. Our findings may offer novel therapeutic strategies for especially patients with SF3B1-mutated LMNs. The role of BAP1 in the pathogenesis of primary LMNs is less clear, immunohistochemistry suggests that BAP1 mutation is infrequent in tumors with disomy for chromosome 3 . However, more detailed analysis is needed for further elucidation of the role of $B A P 1$ in the oncogenesis of primary LMNs. Demonstration of BAP1 mutations in primary LMNs would potentially have therapeutic relevance as well, as epigenetic modulators are now being evaluated in patients with BAP1-mutant UMs. All in all, this study thus underscores the genetic resemblance of primary LMNs and UMs and provides some additional clues for the diagnosis of and therapeutic interference with these tumors.

\footnotetext{
Abbreviations

CNS: Central nervous system; CM: Cutaneous melanoma; FFPE: Formalin fixed and paraffin embedded; HDAC: Histone deacetylase inhibitors; LM: Leptomeningeal; LMN: Leptomeningeal melanocytic neoplasms; UM: Uveal melanoma.

Competing interests

The authors declare that they have no competing interest.

Authors' contribution

$\mathrm{HK}, \mathrm{CP}, \mathrm{WB}, \mathrm{PW}, \mathrm{BK}$, and PG contributed substantially to conception and design of the study. All authors contributed substantially to acquisition of data, and analysis and interpretation of data. HK wrote and edited the
} 
manuscript. All authors contributed in drafting the manuscript and revising it for intellectual content. MJ carried out the molecular genetic analyses. CP supervised the molecular genetic analyses. DC, WW, WB, BK, and HK supervised and scored the BAP1 immunohistochemistry. HK revised histology. All authors read and approved the final version of the manuscript.

\section{Acknowledgments}

We thank Pieter Vandevelde for technical assistance with the lay-out of the figures.

\section{Author details}

'Department of Pathology, Canisius Wilhelmina Hospital, P.O. Box $90156500 G S$, Nijmegen, The Netherlands. 'Department of Pathology, Ghent University Hospital, De Pintelaan 185, 9000 Ghent, Belgium. ${ }^{3}$ Department of Pathology, Radboud University Medical Center, P.O. Box 91016500HB, Nijmegen, The Netherlands. ${ }^{4}$ Department of Pathology, Maastricht University Medical Center, P.O. Box 58006202AZ, Maastricht, The Netherlands. ${ }^{5}$ Department of Pathology, VU University Medical Center, P.O. Box 70571007MB, Amsterdam, The Netherlands.

\section{Received: 6 December 2015 Accepted: 4 January 2016} Published online: 15 January 2016

\section{References}

1. Brat DJ, Perry A. Melanocytic lesions. In: Louis DN, Ohgaki H, Wiestler OD, Cavenee WK, editors. WHO classification of tumours of the central nervous system. Lyon: IARC; 2007. p. 181-3.

2. Kusters-Vandevelde HV, Klaasen A, Kusters B, Groenen PJ, van Engen-van Grunsven IA, van Dijk MR, et al. Activating mutations of the GNAQ gene: a frequent event in primary melanocytic neoplasms of the central nervous system. Acta Neuropathol. 2010;119:317-23. doi:10.1007/s00401-009-0611-3.

3. Van Raamsdonk CD, Bezrookove V, Green G, Bauer J, Gaugler L, O'Brien JM et al. Frequent somatic mutations of GNAQ in uveal melanoma and blue naevi. Nature. 2009:457:599-602. doi:10.1038/nature07586.

4. Murali R, Wiesner T, Rosenblum MK, Bastian BC. GNAQ and GNA11 mutations in melanocytomas of the central nervous system. Acta Neuropathol. 2012:123:457-9. doi:10.1007/s00401-012-0948-x.

5. Gessi M, van de Nes J, Griewank K, Barresi V, Buckland ME, Kirfel J, et al. Absence of TERT promoter mutations in primary melanocytic tumours of the central nervous system. Neuropathol Appl Neurobiol. 2014;40:794-7. doi:10.1111/nan.12138.

6. Dono M, Angelini G, Cecconi M, Amaro A, Esposito Al, Mirisola V, et al. Mutation frequencies of GNAQ, GNA11, BAP1, SF3B1, EIF1AX and TERT in uveal melanoma: detection of an activating mutation in the TERT gene promoter in a single case of uveal melanoma. Br J Cancer. 2014;110:1058-65. doi:10.1038/bjc.2013.804

7. Van Raamsdonk CD, Griewank KG, Crosby MB, Garrido MC, Vemula S, Wiesner T, et al. Mutations in GNA11 in Uveal Melanoma. N Engl J Med. 2010;363:2191-9. doi:10.1059/NEJMoa1000584.

8. Griewank KG, Murali R, Puig-Butille JA, Schilling B, Livingstone E, Potrony M, et al. TERT promoter mutation status as an independent prognostic factor in cutaneous melanoma. J Natl Cancer Inst. 2014;106:doi:10.1093/jnci/dju246.

9. Harbour JW, Onken MD, Roberson ED, Duan S, Cao L, Worley LA, et al. Frequent mutation of BAP1 in metastasizing uveal melanomas. Science. 2010;330:1410-3. doi:10.1126/science.1194472.

10. Ewens KG, Kanetsky PA, Richards-Yutz J, Purrazzella J, Shields CL, Ganguly T, et al. Chromosome 3 status combined with BAP1 and EIF1AX mutation profiles are associated with metastasis in uveal melanoma. Invest Ophthalmol Vis Sci. 2014;55:5160-7. doi:10.1167/iovs.14-14550.

11. Scheuermann JC, de Ayala Alonso AG, Oktaba K, Ly-Hartig N, McGinty RK, Fraterman S, et al. Histone H2A deubiquitinase activity of the Polycomb repressive complex PR-DUB. Nature. 2010;465:243-7. doi:10.1038/nature08966.

12. Koopmans $A E$, Verdijk RM, Brouwer RW, van den Bosch TP, van den Berg $\mathrm{MM}$, Vaarwater J, et al. Clinical significance of immunohistochemistry for detection of BAP1 mutations in uveal melanoma. Mod Pathol. 2014;27: 1321-30. doi:10.1038/modpathol.2014.43.

13. Field MG, Harbour JW. Recent developments in prognostic and predictive testing in uveal melanoma. Curr Opin Ophthalmol. 2014;25:234-9. doi:10. 1097/ICU.0000000000000051.

14. Abdel-Rahman MH, Pilarski R, Cebulla CM, Massengill JB, Christopher BN, Boru G, et al. Germline BAP1 mutation predisposes to uveal melanoma, lung adenocarcinoma, meningioma, and other cancers. J Med Genet. 2011; 48:856-9. doi:10.1136/jmedgenet-2011-100156.

15. Carbone M, Ferris LK, Baumann F, Napolitano A, Lum CA, Flores EG, et al. BAP1 cancer syndrome: malignant mesothelioma, uveal and cutaneous melanoma, and MBAITs. J Transl Med. 2012;10:179. doi:10.1186/1479-5876-10-179.

16. de la Fouchardiere A, Cabaret O, Petre J, Aydin S, Leroy A, de Potter P, et al. Primary leptomeningeal melanoma is part of the BAP1-related cancer syndrome. Acta Neuropathol. 2015;129:921-3. doi:10.1007/s00401-015-1423-2.

17. Martin M, Masshofer L, Temming P, Rahmann S, Metz C, Bornfeld N, et al. Exome sequencing identifies recurrent somatic mutations in EIF1AX and SF3B1 in uveal melanoma with disomy 3. Nat Genet. 2013;45:933-6. doi:10.1038/ng.2674.

18. Harbour JW, Roberson ED, Anbunathan H, Onken MD, Worley LA, Bowcock AM. Recurrent mutations at codon 625 of the splicing factor SF3B1 in uveal melanoma. Nat Genet. 2013;45:133-5. doi:10.1038/ng.2523.

19. Furney SJ, Pedersen M, Gentien D, Dumont AG, Rapinat A, Desjardins L, et al. SF3B1 mutations are associated with alternative splicing in uveal melanoma. Cancer Discov. 2013;3:1122-9. doi:10.1158/2159-8290.cd-13-0330.

20. Kong Y, Krauthammer M, Halaban R. Rare SF3B1 R625 mutations in cutaneous melanoma. Melanoma Res. 2014;24:332-4. doi:10.1097/cmr. 0000000000000071.

21. Casparie M, Tiebosch AT, Burger G, Blauwgeers $H$, van de Pol A, van Krieken $\mathrm{JH}$, et al. Pathology databanking and biobanking in The Netherlands, a central role for PALGA, the nationwide histopathology and cytopathology data network and archive. Cell Oncol. 2007;29:19-24.

22. Kusters-Vandevelde HV, van Engen-van Grunsven IA, Coupland SE, Lake SL, Rijntjes J, Pfundt R, et al. Mutations in g protein encoding genes and chromosomal alterations in primary leptomeningeal melanocytic neoplasms. Pathol Oncol Res. 2015;21:439-47. doi:10.1007/s12253-014-9841-3.

23. Kusters-Vandevelde HV, van Engen-van Grunsven IA, Kusters B, van Dijk MR, Groenen PJ, Wesseling P, et al. Improved discrimination of melanotic schwannoma from melanocytic lesions by combined morphological and GNAQ mutational analysis. Acta Neuropathol. 2010;120:755-64. doi:10.1007/s00401-010-0749-z.

24. van Dongen JJ, Langerak AW, Bruggemann M, Evans PA, Hummel M, Lavender $\mathrm{FL}$, et al. Design and standardization of PCR primers and protocols for detection of clonal immunoglobulin and T-cell receptor gene recombinations in suspect lymphoproliferations: report of the BIOMED-2 Concerted Action BMH4-CT98-3936. Leukemia. 2003;17:2257-317. doi:10.1038/sj.leu.2403202.

25. van Engen-van Grunsven AC, Baar MP, Pfundt R, Rijntjes J, KustersVandevelde HV, Delbecq AL, et al. Whole-genome copy-number analysis identifies new leads for chromosomal aberrations involved in the oncogenesis and metastastic behavior of uveal melanomas. Melanoma Res. 2015:25:200-9. doi:10.1097/CMR.0000000000000152.

26. Kalirai H, Dodson A, Faqir S, Damato BE, Coupland SE. Lack of BAP1 protein expression in uveal melanoma is associated with increased metastatic risk and has utility in routine prognostic testing. Br J Cancer. 2014;111:1373-80. doi:10.1038/bjc.2014.417.

27. Shah AA, Bourne TD, Murali R. BAP1 protein loss by immunohistochemistry: a potentially useful tool for prognostic prediction in patients with uveal melanoma. Pathology. 2013;45:651-6. doi:10.1097/pat.0000000000000002.

28. van Essen TH, van Pelt SI, Versluis M, Bronkhorst IH, van Duinen SG, Marinkovic M, et al. Prognostic parameters in uveal melanoma and their association with BAP1 expression. Br J Ophthalmol. 2014;98:1738-43. doi:10.1136/bjophthalmol-2014-305047.

29. Landreville S, Agapova OA, Matatall KA, Kneass ZT, Onken MD, Lee RS, et al. Histone deacetylase inhibitors induce growth arrest and differentiation in uveal melanoma. Clin Cancer Res. 2012;18:408-16. doi:10.1158/10780432.ccr-11-0946.

30. Yoshida K, Ogawa S. Splicing factor mutations and cancer. Wiley Interdiscip Rev RNA. 2014:5:445-59. doi:10.1002/wrna.1222.

31. Biankin AV, Waddell N, Kassahn KS, Gingras MC, Muthuswamy LB, Johns AL, et al. Pancreatic cancer genomes reveal aberrations in axon guidance pathway genes. Nature. 2012;491:399-405. doi:10.1038/nature11547.

32. Maguire SL, Leonidou A, Wai P, Marchio C, Ng CK, Sapino A, et al. SF3B1 mutations constitute a novel therapeutic target in breast cancer. J Pathol. 2015;235:571-80. doi:10.1002/path.4483.

33. Wang L, Lawrence MS, Wan Y, Stojanov P, Sougnez C, Stevenson K, et al. SF3B1 and other novel cancer genes in chronic lymphocytic leukemia. N Engl J Med. 2011;365:2497-506. doi:10.1056/NEJMoa1109016. 
34. Malcovati L, Papaemmanuil E, Bowen DT, Boultwood J, Della Porta MG, Pascutto $\mathrm{C}$, et al. Clinical significance of SF3B1 mutations in myelodysplastic syndromes and myelodysplastic/myeloproliferative neoplasms. Blood. 2011; 118:6239-46. doi:10.1182/blood-2011-09-377275.

35. Pedersen M, Kusters-Vandevelde HV, Viros A, Groenen PJ, Sanchez-Laorden $\mathrm{B}$, Gilhuis $\mathrm{JH}$, et al. Primary melanoma of the CNS in children is driven by congenital expression of oncogenic NRAS in melanocytes. Cancer Discov. 2013;3:458-69. doi:10.1158/2159-8290.CD-12-0464.

36. Kinsler VA, Thomas AC, Ishida M, Bulstrode NW, Loughlin S, Hing S, et al. Multiple congenital melanocytic nevi and neurocutaneous melanosis are caused by postzygotic mutations in codon 61 of NRAS. J Invest Dermatol. 2013;133:2229-36. doi:10.1038/jid.2013.70.

37. Kusters-Vandevelde HV, Kusters B, van Engen-van Grunsven AC, Groenen PJ, Wesseling P, Blokx WA. Primary melanocytic tumors of the central nervous system: a review with focus on molecular aspects. Brain Pathol. 2015;25: 209-26. doi:10.1111/bpa.12241.

38. Hunter SM, Anglesio MS, Ryland GL, Sharma R, Chiew YE, Rowley SM, et al. Molecular profiling of low grade serous ovarian tumours identifies novel candidate driver genes. Oncotarget. 2015;6:37663-77. doi:10.18632/ oncotarget.5438.

39. Kunstman JW, Juhlin CC, Goh G, Brown TC, Stenman A, Healy JM, et al. Characterization of the mutational landscape of anaplastic thyroid cancer via whole-exome sequencing. Hum Mol Genet. 2015;24:2318-29. doi:10.1093/hmg/ddu749.

40. Chaudhuri J, Si K, Maitra U. Function of eukaryotic translation initiation factor 1A (elF1A) (formerly called elF-4C) in initiation of protein synthesis. J Biol Chem. 1997;272:7883-91.

Submit your next manuscript to BioMed Central and we will help you at every step:

- We accept pre-submission inquiries

- Our selector tool helps you to find the most relevant journal

- We provide round the clock customer support

- Convenient online submission

- Thorough peer review

- Inclusion in PubMed and all major indexing services

- Maximum visibility for your research

Submit your manuscript at www.biomedcentral.com/submit
Biomed Central 